\title{
A SOFTWARE CONSULTING SERVICE FOR NETWORK USERS
}

As actual or attempted Network use has grown, it has become increasingly apparent that users with real problems to solve are not afforded the kinds of software assistance and consulting services normally provided by computer service facilities. Two mechanisms which were designed to help fill the void in the past are the Resources Notebook and the designation of Techincal Liaisons. Unfortunately, the Resources Notebook tends to have a long undate cycle, and Technical Liaisons are frequently systems programmers who may work at odd hours and are sometimes impatient with the questions of naive users.

In an attempt to deal with this problem in a more satisfactory way, BBN recently aided a "software consultant", Nancy Neigus, to the Networtk Control Center staff. Nancy has extensive software experience on a number of systems, and has spent the last few months familiarizing with server systems and subsystems via the network.

This consulting service is experimental in nature, and as such its limits, even its continued existance, will be influenced by the expressed needs of the user community. Altough the primary goal is for Nancy to provide assistan ce in using existing resources, we do not (currently) exclude the possibility of her participation in such projects as the checkout of protocol implementations and resolution of any discovered conflicts. In some cases, it may turn out that successful network operation of a subsystem requires changes to Host software; we will attempt to act as a repository and dispatch point of reported difficulties of its kind. telephone:

Nancy can be contacted via the Network Control Center

(617) 611-0100

Collect calls will be accepted. She will normally be available between $9 \mathrm{am}$ and $5 \mathrm{pm}$ (Eastern Time) weekdays, but the NCC has round-the-clock operator coverage and the operators will take messages for her at other times. Online messages may also be left at the NIC for "NJN", or, for TIP users, vis the "Gripe" service (see TIP news).

Please inform users at your site of the this software consulting service, and encourage them to try it.

[ This RFC was put into machine readable form for entry ]

[ into the online RFC archives by Chakravarthy Paila 4/97] 